\title{
Temperature-dependent Thermal Stress Determination for Through-Silicon-Vias (TSVs) by Combining Bending Beam Technique with Finite Element Analysis
}

\author{
Kuan H. Lu, Suk-Kyu Ryu*, Qiu Zhao, Klaus Hummler**, Jay Im, Rui Huang*, and Paul S. Ho \\ Microelectronics Research Center, University of Texas at Austin, Austin, TX 78758 \\ *Department of Aerospace Engineering and Engineering Mechanics, University of Texas at Austin, Austin, Texas 78712 \\ **siXis Inc., Research Triangle Park, NC 27709 \\ kuan.h.lu@gmail.com
}

\begin{abstract}
In this paper, temperature-dependent thermal stresses in $\mathrm{Cu}$ TSVs are measured by combining the bending beam experiment with a finite element analysis (FEA). The bending beam technique measures the averaged bending curvature induced by the thermal expansion of a periodic annular $\mathrm{Cu}$ TSV array. The structural complexity of the blind annular TSV necessitated the use of FEA to derive the TSV-induced thermal stresses which accounts for the beam bending during thermal cycles. The FEA simulations established linear relationships between bending curvature and stress components in TSVs. Such linear relationships were used to extract independent stress components from the bending beam measurements. The results provided an understanding to the temperature-dependent stress characteristics in TSVs.
\end{abstract}

\section{Introduction}

In 3-D ICs, the through-silicon-via (TSV) is a critical element connecting die-to-die in the integrated stack structure. The thermal expansion mismatch between the TSVs and the $\mathrm{Si}$ matrix can induce sufficient stresses to drive interfacial crack growth and to degrade the device performance in 3-D ICs [1][2][3]. This raises serious reliability concerns and has stimulated great interests from the semiconductor industry to investigate the thermal stress characteristics and their impact on the structural reliability of TSV structures. The stress state of TSV is highly complex and three-dimensional in nature owing to its high-aspect ratio and the design of the 3-D stack structure. To study the stress state of such complexity, it is important to understand the nature of the thermal stresses and their dependence on the material, configuration, and processing of the TSV structures. To investigate the thermal stress characteristics, two general approaches are usually taken in a complimentary fashion: one based on experimental measurements and the other by analytical methods or modeling simulations.

Recently, researchers for 3-D interconnects have been developing several stress measurement techniques to characterize TSV-induced thermal stresses [1][4][5]. For example, the micro-Raman technique has demonstrated its capability in measurement of the stress distribution in $\mathrm{Si}$ around individual TSVs with a sub-micron spatial resolution. Such a technique, usually operated at room temperature, measures the sum of normal stress components near the $\mathrm{Si}$ wafer surface. To extract individual stress components, therefore, finite-element-analysis (FEA) is required [4].

In this study, we developed a metrology to determine temperature-dependent 3-D thermal stresses in TSV structures by combining the bending beam technique [6] with FEA simulations. TSV wafers containing periodic arrays of blind annular $\mathrm{Cu}$ TSVs are designed and fabricated, and the bending beam specimens are diced from these TSV wafers into $4 \mathrm{~mm}-$ by-40 mm strips. Such test specimens are thermal-cycled in a vacuum chamber, and the resulting bending curvature is monitored using a laser-positioning system. The bending curvature provides a measure of the global thermal stresses in the composite TSV structure. Then, FEA simulations are performed to correlate the beam curvature to the stress components in the TSV test structure. The simulations show that the stresses in the TSV structures are linearly dependent on the bending curvature of the Si beams. Finally, the 3-D thermal stresses in the local level around individual TSVs are derived by combining the observed bending curvature with the FEA simulations. The thermal stresses in $\mathrm{Si}$ and in annular $\mathrm{Cu}$ TSVs both exhibit a stress hysteresis behavior under thermal cycles, owing to the plasticity of electroplated $\mathrm{Cu}$ material.

\section{Curvature measurement using bending beam system}

The bending beam technique for thermal stress measurement is introduced in this section. Here only the experimental procedures are explained while the FEA simulations to deduce the thermal stresses in an annular TSV structure is described in later sections of this paper. The bending beam system employs an optical method to monitor the curvature of TSV specimens with respect to temperature. A schematic setup of the bending beam system is shown in Figure 1. The whole system consists of a laser-positioning module, a thermal cycling vacuum chamber, and a controlling computer.

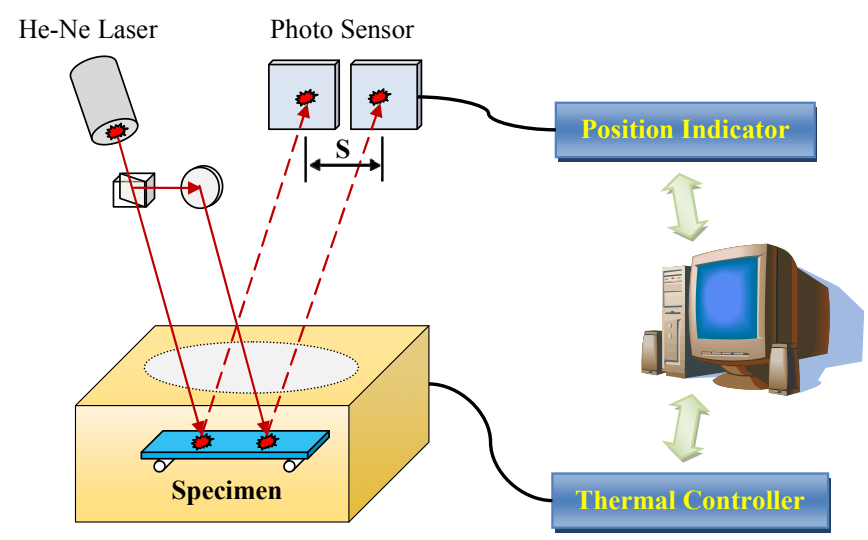

Heating Block in Vacuum Chamber

Figure 1. Schematic setup of the bending beam system.

As illustrated in Figure 1, specimens of a strip shape are placed on a heating stage in the vacuum chamber. To measure the bending curvature of the specimen, the He-Ne laser beam 
is split into two parts with each impinging on the surface of the specimen through a transparent viewing window, and then the positions of the reflected laser beams are tracked using two photo sensors. The distance between the two laser spots on photo sensors, $S$, is measured using the laser-positioning module. The bending curvature, $\kappa$, can be expressed as below [7]:

$$
\kappa-\kappa_{o}=\frac{1}{R}-\frac{1}{R_{o}}=\frac{S_{o}-S}{2 D L}
$$

where $R$ is the radius of curvature, $D$ is the distance between the two laser spots on the specimen, and $L$ is the distance between the specimen and photo sensors. The subscript " $o$ " stands for the baselines of a reference state. The spatial relationships among these parameters are illustrated in Figure 2 .

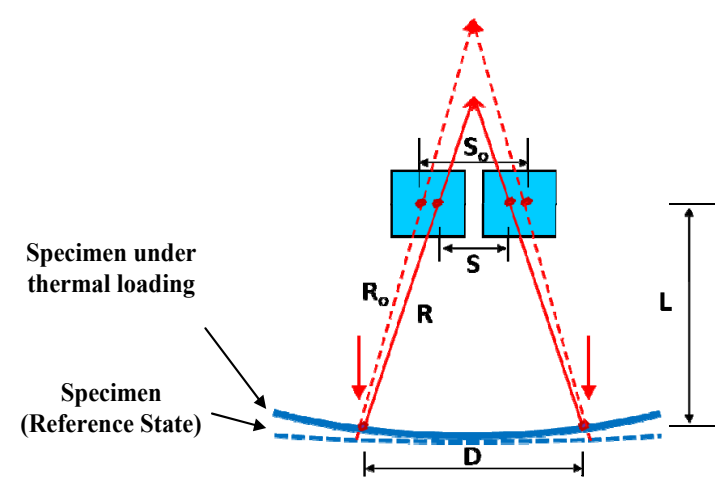

Figure 2. Spatial relationships among parameters in Equation 1.

\section{Bending beam specimen preparation: annular $\mathrm{Cu}$ via array in $\mathrm{Si}$}

Si wafers containing periodic arrays of blind annular $\mathrm{Cu}$ TSVs were co-designed by UT and siXis Inc. for bending beam measurement. To fabricate the annular vias, a square grid array of circular areas ( $90 \mu \mathrm{m}$ in diameter) is first photopatterned on (001) Si wafers, and then a Bosch Si etching process is performed to drill circular holes to form periodic blind vias on the $\mathrm{Si}$ wafer. After the $\mathrm{Si}$ etching process, a conformal coating of dielectric material $(\sim 2 \mu \mathrm{m}$ thick silicon oxide) is deposited on the wafer surface and at the via sidewall. Finally, the wafers are electroplated with a thin layer of $\mathrm{Cu} \sim 2 \mu \mathrm{m}$ in thickness to form annular $\mathrm{Cu}$ vias.

The samples used for bending beam measurements are diced from the Si wafer into $4 \mathrm{~mm}$-by- $40 \mathrm{~mm}$ strips. In order to measure the thickness of $\mathrm{Cu}$ on the via sidewalls, the bending beam specimens are polished on the edge to expose the cross-section of a row of TSVs. A cross-section SEM image of the annular via array is shown in Figure 3.

The electroplated annular $\mathrm{Cu}$ layer does not cover the whole length of the via sidewalls. As shown in Figure 3, the electroplated $\mathrm{Cu}$ film covers about upper two thirds of the via sidewalls. The dimensions of annular vias are measured from the cross-section of the specimen using a SEM/FIB system. Table 1 lists the averaged height and thickness of the annular via structure.

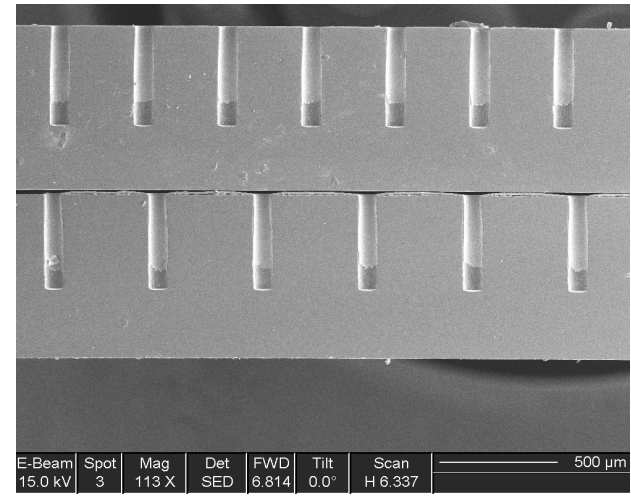

Figure 3. Cross-section image of bending beam specimen.

Table 1. Bending beam sample dimension.

\begin{tabular}{|c|c|}
\hline Annular via array $\left(\mathrm{D}_{\mathrm{f}}: 90 \mu \mathrm{m}\right.$, pitch: $\left.360 \mu \mathrm{m}\right)$ & Unit: $\mu \mathrm{m}$ \\
\hline Thickness of Wafer & $710 \pm 3$ \\
\hline Height of via hole & $429 \pm 5$ \\
\hline Height of Cu via & $311 \pm 7$ \\
\hline Thickness of annular Cu via & $1.6 \pm 0.3$ \\
\hline Thickness of silicon oxide & $2.0 \pm 0.2$ \\
\hline
\end{tabular}

To complete the TSV test structure, the overburden layers of silicon oxide and $\mathrm{Cu}$ on the wafer surface are mechanically polished off. The top Si surface is exposed after polishing. The finished bending beam specimen is illustrated in Figure 4.

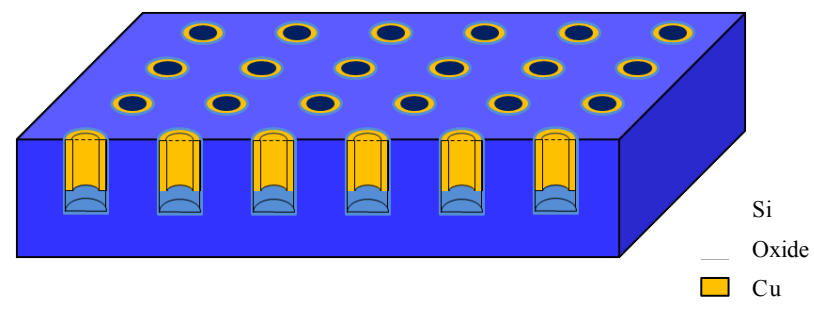

Figure 4. Visual illustration of bending beam specimen.

\section{Bending beam experiment and results}

After the sample preparation, a specimen containing vias of $90 \mu \mathrm{m}$ in diameter is selected for the bending beam experiment. The pitch size between adjacent vias is $360 \mu \mathrm{m}$. The specimen is placed in a vacuum test chamber with a 100 Torr of ambient dry nitrogen. The specimen is placed upside down on a supporting stage so that the incident laser beams are reflected by the backside of the specimen, i.e. the side without exposed vias. If the front side (i.e. the side containing via array) is selected as the reflecting surface, incident laser beams will be diffracted by the periodic via array, yielding multiple diffracted laser spots on the photo sensors and false signals in the position indicator.

To start the stress measurement, the bending beam specimen is thermal-cycled between the room temperature and $200^{\circ} \mathrm{C}$ in the vacuum chamber. The bending curvature of the specimen is continuously monitored as a function of temperature. Several factors contribute to the bending of the specimen: (a) the thermal expansion of $\mathrm{Cu}$ vias, (b) the thermal expansion of silicon oxide liner, and (c) the initial 
curvature of the bare $\mathrm{Si}$ beam. To extract the thermal stress contribution of the $\mathrm{Cu}$ vias to the beam bending, the baseline curvatures induced by (b) and (c) have to be subtracted out from the overall bending. For this purpose, $\mathrm{Cu}$ vias are etched off from the test specimen using a nitric acid solution, and the baseline curvature is measured again under the same thermal cycles. By subtracting off the baseline curvature, the bending induced by the thermal expansion of the $\mathrm{Cu}$ via array is deduced.

Following this procedure, the bending curvature induced by $\mathrm{Cu}$ vias is obtained, and the result is plotted in Figure 5 as a function of temperature. The result shows clearly that the deformation of the $\mathrm{Cu}$ vias exhibits hysteresis phenomenon during two thermal cycles between $30^{\circ} \mathrm{C}$ and $200^{\circ} \mathrm{C}$. Since $\mathrm{Si}$ and silicon oxide are both linear elastic in nature, the hysteresis behavior can be attributed to plasticity of the $\mathrm{Cu}$ layer in the via. This will be further discussed in the next section when the observed bending curvature in Figure 5 is analyzed to deduce the stress characteristics of the via structure.

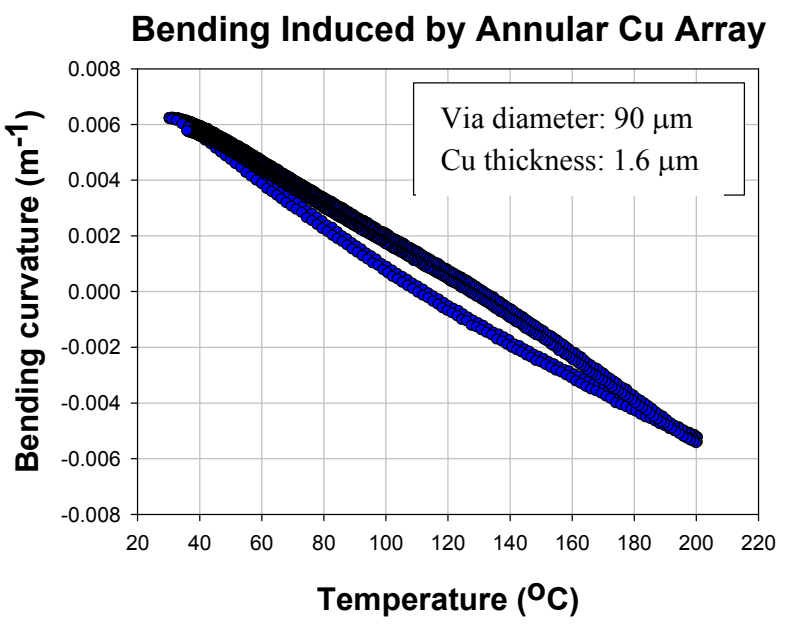

Figure 5. Bending curvature induced by periodic annular $\mathrm{Cu}$ array under repeated thermal cycles between $30^{\circ} \mathrm{C}$ to $200^{\circ} \mathrm{C}$.

\section{Thermal stress analysis for annular Cu TSVs}

In this study, the thermal stress characteristics are deduced from the bending curvature of the $\mathrm{Cu}$ via structures observed during thermal cycling. First, an analogous example of bending beam stress measurement on a thin film is described as following. Consider an isotropic thin film lying on top of a thick Si substrate. The thermal stress on the thin film as a result of thermal loading is equal-biaxial and 2-D where there is no out-of-plane stress. Assuming that the thin film thickness $h_{f}$ is much thinner than the substrate thickness $h_{s}$, the equibiaxial stress of the thin film, $\sigma_{f}$, is proportional to the net curvature induced by the film, $\kappa-\kappa_{o}$ :

$$
\sigma_{f}=\frac{B_{s} h_{s}^{2}}{6 h_{f}}\left(\kappa-\kappa_{o}\right)
$$

where $B_{s}$ is the biaxial modulus of the substrate. Equation 2 is the well-known Stoney's equation [8].
Now consider the case of annular $\mathrm{Cu}$ vias embedded in the $\mathrm{Si}$ matrix (Figure 4). Inside an annular $\mathrm{Cu}$ via, the thermal expansion mismatch between $\mathrm{Cu}$ and the $\mathrm{Si}$ matrix can induce thermal stresses during thermal cycling along the radial direction $\left(\sigma_{r r}\right)$, the circumferential direction $\left(\sigma_{\theta \theta}\right)$, and the axial direction $\left(\sigma_{z z}\right)$. The stress state in such a $\mathrm{Cu}$ via is 3-D in nature, and the bending curvature of the whole $\mathrm{Si}$ beam is induced by the radial stress $\sigma_{r r}$ and the circumferential stress $\sigma_{\theta \theta}$. Due to the complex geometry, the stress components cannot be deduced simply from the bending curvature based on Equation 2. Therefore, an FEA is used to calculate the stress behavior of the Cu TSV test beam and to establish the relationship between the bending curvature $\kappa$ and the stress components during thermal cycling. Based on the observed bending curvature, the stress components of the $\mathrm{Cu}$ via can be deduced. The FEA simulation procedure is described in the following section.

\section{FEA simulation for annular Cu TSVs}

The thermal stress-induced bending curvature is simulated using a commercial software $\left(\right.$ ANSYS $^{\circledR}$ ). A simplified FEA model containing a 5-by-2 square grid annular $\mathrm{Cu}$ via array is constructed, as illustrated in Figure 6a. The geometrical dimensions of $\mathrm{Cu}$ vias in this model are listed in Table 1. Note that the height of annular $\mathrm{Cu}$ vias is less than half the thickness of $\mathrm{Si}$ wafer (Figure 3). A 3-D solid element (SOLID185) in the ANSYS software is selected for simulations. The elemental mesh in one of the ten unit cells is shown in Figure 6b, while the elemental mesh of the annular $\mathrm{Cu} /$ silicon oxide is magnified and shown in Figure $6 \mathrm{c}$. The thermo-mechanical properties of materials used for simulations are listed in Table 2. Among them, the CTE of silicon oxide is intentionally assumed to be the same as that of $\mathrm{Si}$. By excluding the CTE mismatch between silicon oxide and the Si substrate, the beam bending due to the thermal expansion of $\mathrm{Cu}$ material is simulated and is to be compared with the experimental results in the next section.

A symmetric boundary condition is applied on the surfaces at $x=0$ and at $y=0$. The FEA model is simulated under varying thermal load $\Delta \mathrm{T}\left(-50^{\circ} \mathrm{C} \sim-200^{\circ} \mathrm{C}\right)$. After the simulation is complete, the bending curvature and the stress components are extracted from the result files for analysis.

Table 2. Thermo-mechanical properties of materials $[9,10,11]$

\begin{tabular}{|c|c|c|c|}
\hline Material & CTE $(\mathrm{ppm} / \mathrm{K})$ & $\begin{array}{c}\text { Young's } \\
\text { Modulus }(\mathrm{GPa})\end{array}$ & Poisson's ratio \\
\hline $\mathrm{Si}$ & 2.3 & 130 & 0.28 \\
\hline $\mathrm{Cu}$ & 17 & 110 & 0.35 \\
\hline Silicon oxide & $2.3^{1}$ & 76 & 0.18 \\
\hline
\end{tabular}

\section{Thermal stresses on top Si surface}

The thermal stresses on the top $\mathrm{Si}$ surface are first analyzed. In a cylindrical coordinate system, the stress components are axi-symmetric. According to the semianalytical near-surface solution [2], the radial stress $\sigma_{r r}$ and the circumferential stress $\sigma_{\theta \theta}$ on the top Si surface (Figure 6a, $z=0$ ) are of the same magnitude but opposite in sign. The out-of-plane stress component $\sigma_{z z}$ vanishes on the surface.

${ }^{1}$ The CTE of silicon oxide is intentionally assumed to be the same as that of Si. 
Thus, only the radial stress from FEA simulation is examined here. The distribution of $\sigma_{r r}$ on the top surface obtained by FEA is plotted as a function of the radial distance $r$ in Figure 7. Under thermal load $\Delta \mathrm{T}$, the bending curvature of the FEA model increases monotonically with the increasing thermal load. The radial stress distribution also increases uniformly with the bending curvature on the top Si surface.

(a)

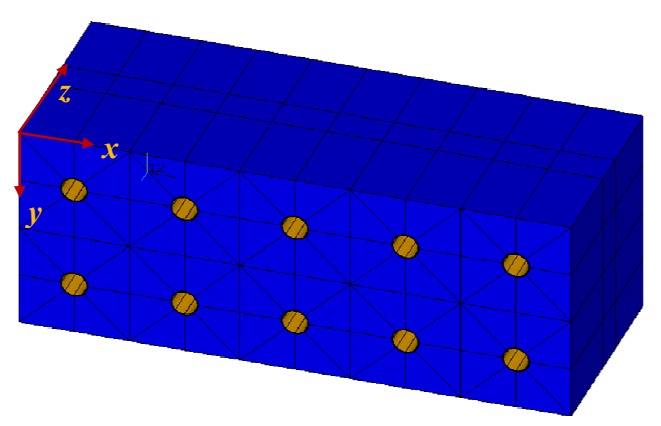

(b)

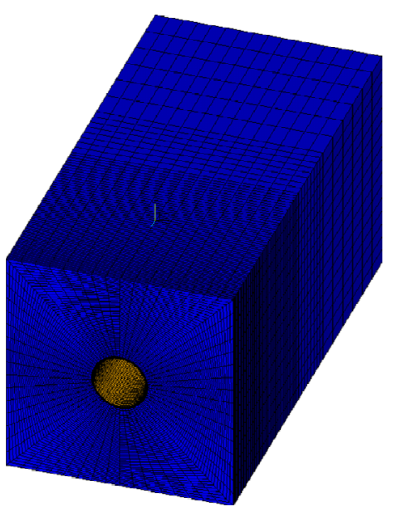

(c)

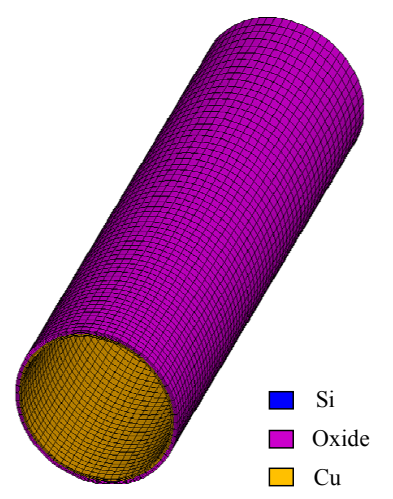

Figure 6. (a) FEA model containing a 5-by-2 annular $\mathrm{Cu}$ via array. (b) Element mesh in one via unit cell. (c) Element mesh in annular $\mathrm{Cu}$ via and the silicon oxide liner.

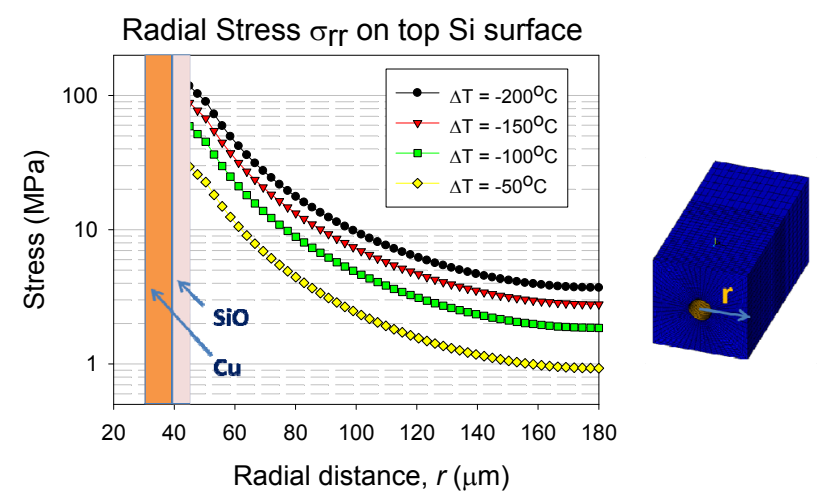

Figure 7. Distribution of radial stress $\sigma_{r r}$ on the top Si surface with varying thermal load $\Delta \mathrm{T}$.

To show that the radial stresses in Figure 7 are proportional to the bending curvature, the simulation result at the TSV/Si boundary on the top surface $(z=0)$ is selected and plotted against the curvature (Figure 8). It exhibits a linear relationship between the radial stress and the bending curvature. Therefore, the linear curve-fit equation shown in
Figure 8 can be used to obtain the corresponding radial stress once the bending curvature of the test beam is experimentally determined.

\section{Radial Stress vs. Bending Curvature}

(Si/oxide interface, $z=0$ )

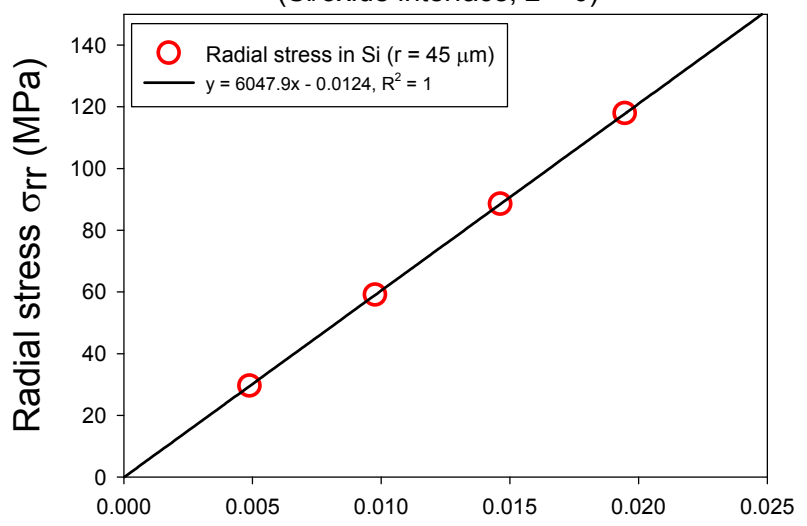

Bending curvature $\left(\mathrm{m}^{-1}\right)$

Figure 8. A linear relationship between the bending curvature and the radial stress $\sigma_{r r}$ at a specific location (top $\mathrm{Si}$ surface, Si/oxide interface).

After establishing the stress-curvature relationship using the FEA, the bending curvature from the experiment is converted to the radial stress for the selected location $(r=$ $\left.\mathrm{D}_{\mathrm{f}} / 2, z=0\right)$. This corresponds to the circumference of the $\mathrm{Cu}$ TSV on the top Si surface where the radial stress can be deduced from the measured beam curvature (Figure 5) and the result is plotted in Figure 9 as a function of temperature for two repeated thermal cycles. As shown in Figure 9, the maximum radial stress $\sigma_{r r}$ in $\mathrm{Si}$ is within a range of $39 \mathrm{MPa}$ (at $30^{\circ} \mathrm{C}$ ) to $-33 \mathrm{MPa}\left(\right.$ at $200^{\circ} \mathrm{C}$ ). The bending beam specimen has a stress-free temperature of $112^{\circ} \mathrm{C}$ or $125^{\circ} \mathrm{C}$, depending on whether a positive or negative thermal load is applied. The non-linear behavior of stress-temperature curve results from the plasticity of $\mathrm{Cu}$ material during thermal cycles, which is discussed in the next section.

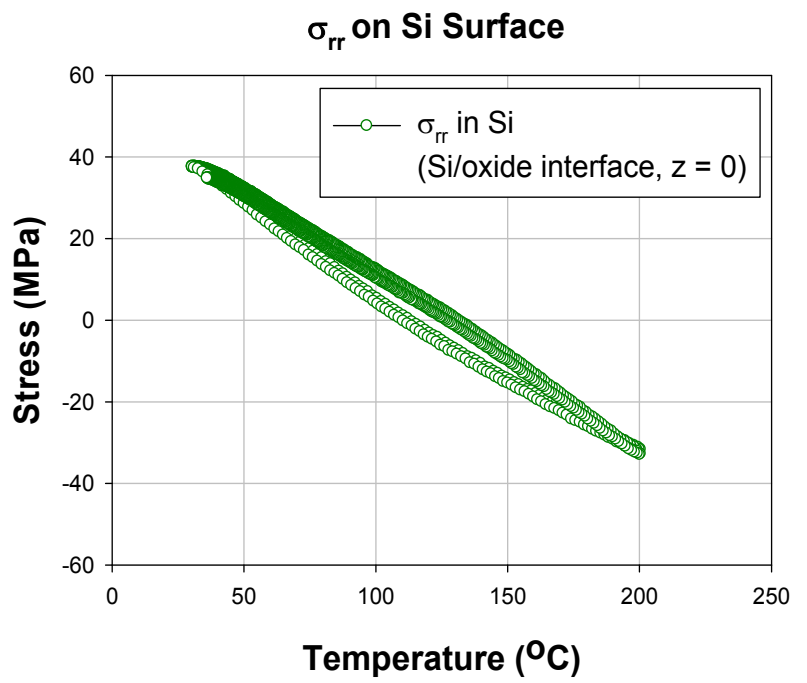

Figure 9. Radial stress $\sigma_{r r}$ vs. temperature (top Si surface, Si/silicon oxide interface). 


\section{Thermal stresses in annular Cu TSVs}

Here the FEA-simulated thermal stresses in an annular $\mathrm{Cu}$ via are analyzed. In a cylindrical coordinate system, the thermal stress distribution in the annular $\mathrm{Cu}$ via is also axisymmetric. Under a thermal load $\Delta \mathrm{T}=-100^{\circ} \mathrm{C}$, thermal stresses in $\mathrm{Cu}$ along the $\mathrm{Cu} /$ oxide interface are plotted as a function of depth $z$ in Figure 10.

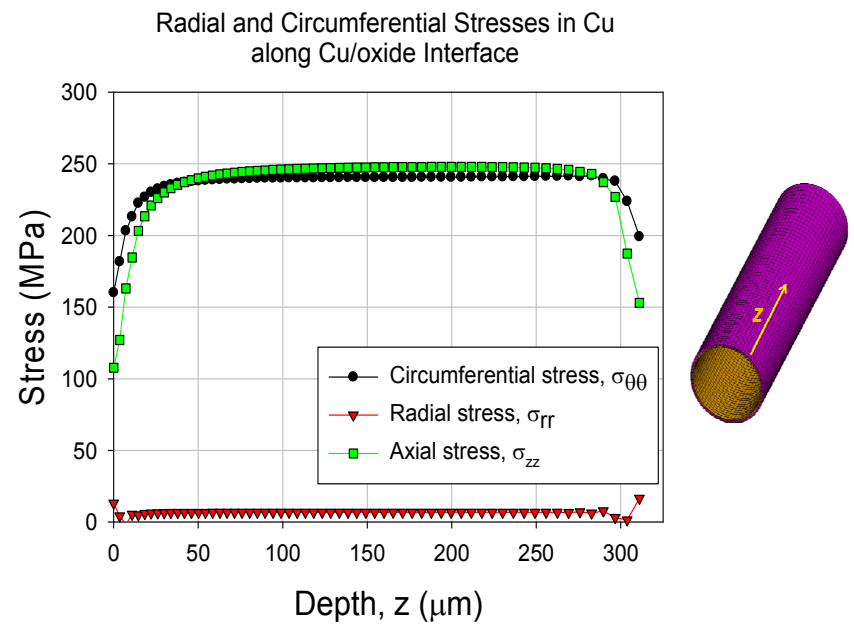

Figure 10. Stresses in annular $\mathrm{Cu}$ as a function of depth $z(\mathrm{Cu} /$ oxide interface, $\left.\mathrm{D}_{\mathrm{f}}=90 \mu \mathrm{m}\right)$.

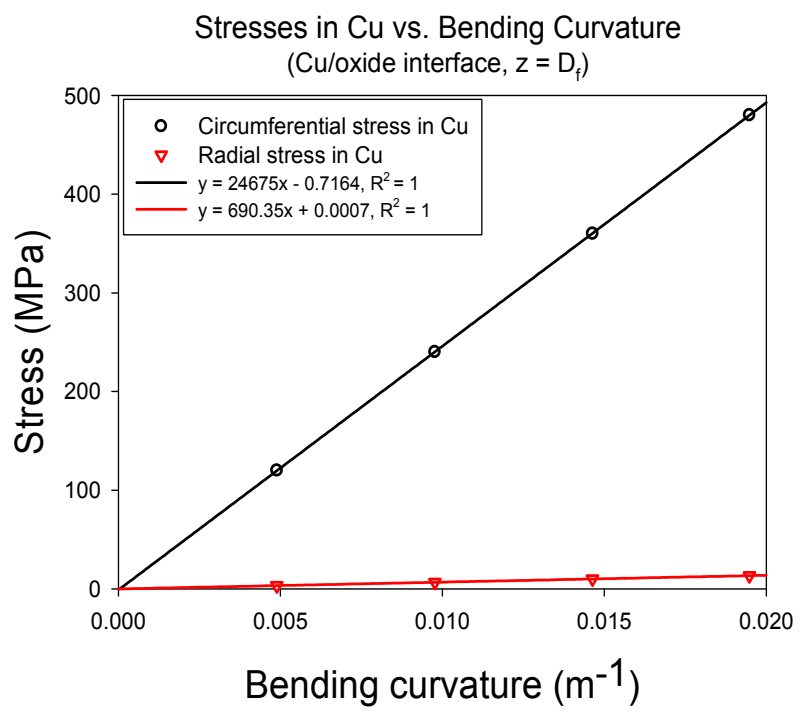

Figure 11. Linear relationship between the bending curvature and the circumferential stress $\sigma_{\theta \theta}$ and the radial stress $\sigma_{r r}$ in annular $\mathrm{Cu}$ via $(\mathrm{Cu} /$ oxide interface $)$.

As shown in Figure 10, the circumferential stress $\sigma_{\theta \theta}$ and the axial stress $\sigma_{z z}$ are of a similar magnitude, but the radial stress $\sigma_{r r}$ is smaller by a factor of 20 . This can be attributed to the fact that the thickness of annular $\mathrm{Cu}$ via is much thinner than the via diameter $\mathrm{D}_{\mathrm{f}}$ so that $\sigma_{r r}$ is equivalent to the normal stress in a thin $\mathrm{Cu}$ film along the out-of-plane direction. It is interesting to note the stress variation as a function of the depth where the stresses approach a steady-state at a distance $\sim D_{f}$ away from both ends of the $\mathrm{Cu}$ cylinder. Therefore, over most of the annular $\mathrm{Cu}$ via, except near the ends, the stress state may be approximated by a 2-D plane-strain solution for a thin-wall cylinder.

In the following, the stress components which contribute to beam bending $\left(\sigma_{\theta \theta}\right.$ and $\left.\sigma_{r r}\right)$ are chosen for analysis. FEA results at a depth $z=\mathrm{D}_{\mathrm{f}}$ on the $\mathrm{Cu}$ /oxide interface are selected for this discussion. FEA-simulated thermal stresses in $\mathrm{Cu}$ are plotted as a function of the bending curvature in Figure 11. Linear equations between stresses and the bending curvature are obtained by curve fitting.

The stresses in annular $\mathrm{Cu}$ vias at $z=\mathrm{D}_{\mathrm{f}}$ can be deduced based on the linear relations established by FEA simulations (Figure 11) and the bending curvature observed (Figure 5). It should be pointed out that such linear relationships between the stresses and the bending curvature applies no matter whether the $\mathrm{Cu}$ material has been mechanically yielded or not. The bending curvature of the $\mathrm{Si}$ beam originates from the force balance between the $\mathrm{Si}$ beam and the via structures on top. Because the Si material is linear elastic, the force (or stress) required to bend the Si beam is linearly proportional to the bending curvature.

The radial and circumferential stresses at the chosen location $\left(r=\mathrm{D}_{\mathrm{f}} / 2-\mathrm{t}_{\text {oxide }}, z=\mathrm{D}_{\mathrm{f}}\right)$ are plotted as a function of temperature in Figure 12.

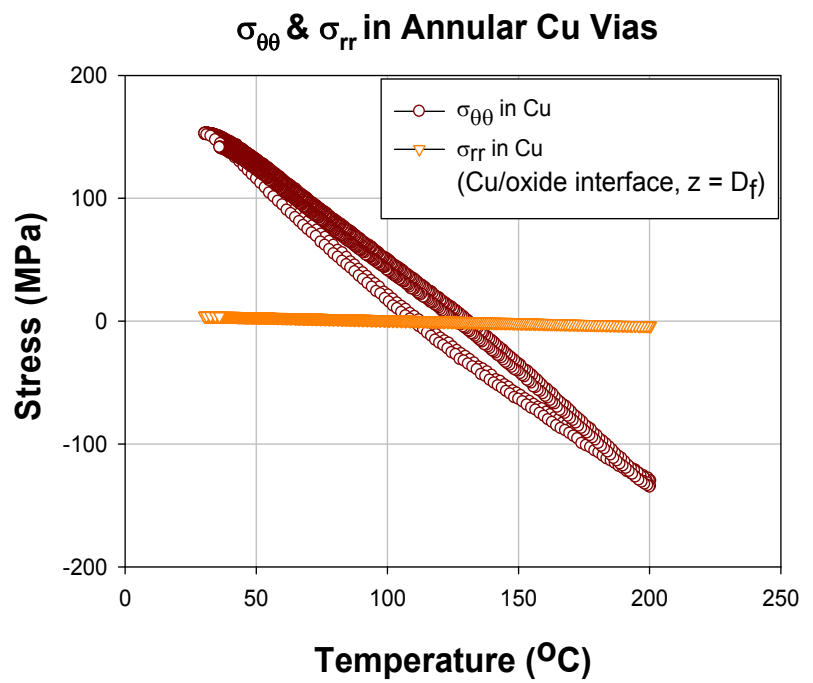

Figure 12. Stress-temperature curves of annular $\mathrm{Cu}$ vias $(\mathrm{Cu} /$ oxide interface).

In Figure 12, the radial stress $\sigma_{r r}$ in the annular $\mathrm{Cu}$ via is almost zero. It is expected because of the traction-free surface on the inner sidewall of the via. The circumferential stress $\sigma_{\theta \theta}$, which is much larger than the radial stress, ranges from +150 $\mathrm{MPa}\left(\right.$ at $30^{\circ} \mathrm{C}$ ) to $-130 \mathrm{MPa}$ (at $200^{\circ} \mathrm{C}$ ). It implies that only the circumferential stress (or force) in $\mathrm{Cu}$ vias accounts for the specimen bending, because the other stress component $\sigma_{r r}$ is a small quantity and $\sigma_{z z}$, although of a larger quantity, does not contribute to bending. The other observation from Figure 12 is the elastic hysteresis behavior of $\mathrm{Cu}$. The annular $\mathrm{Cu}$ via is subjected to a thermal load followed by a reverse thermal load. During the reverse loading (i.e. cooling), the annular $\mathrm{Cu}$ via exhibits an initial elastic unloading behavior, followed by a so called "early yielding" occurring at a stress level below $50 \mathrm{MPa}$. Early yielding is often observed in thermal stress measurements for metallic thin films, which is similar to the 
Bauschinger effect in bulk metals. Dislocation theories were applied in the past to explain such early yielding behavior of $\mathrm{Cu}$ thin films [12]. Because the annular $\mathrm{Cu}$ via investigated here is only $\sim 1.6 \mu \mathrm{m}$ thick, the annular $\mathrm{Cu}$ via is equivalent to a $\mathrm{Cu}$ thin film. The observed "early yielding" behavior may be the same as that of $\mathrm{Cu}$ thin films.

\section{Summary}

A stress measurement on $\mathrm{Cu}$ via arrays using bending beam technique has been demonstrated in this paper. The state-of-the-art curvature measurement technique has a sensitive resolution of $10^{-3} \mathrm{~m}^{-1}$ in curvature, making it possible to detect the beam deflection induced by a small magnitude of stress in $\mathrm{Si}(<50 \mathrm{MPa})$. It has been found challenging to measure the stresses in such annular via structure using other techniques such as micro-Raman.

Bending beam technique can also be used to observe the plasticity behavior of $\mathrm{Cu}$ material during thermal cycling. In Figure 12, a non-linear elastic behavior has been observed in the electroplated $\mathrm{Cu}$ material, and the stress-free temperature of $\mathrm{Cu}$ vias can be determined from such stress-temperature curve. Compared to micro-Raman measurements, which are often operated at room temperature, the bending beam technique provides useful temperature-dependent information for thermo-mechanical analyses. However, the major disadvantage of the bending beam method is the sample configuration, which requires a large population of periodically arranged vias. The other disadvantage is that only averaged global stresses/strains of the whole beam are measured. Such a technique cannot measure stresses near individual vias as micro-Raman does. The local stresses in individual vias are subjected to vary with respect to $\mathrm{Cu}$ thickness, oxide thickness, the elastic anisotropy in $\mathrm{Cu}$ grains, etc. The precise local stresses induced by a specific TSV cannot be obtained using FEA simulations with only averaged sample geometry and with isotropic material properties. Instead, TSV-induced local stresses near the Si surface can be measured using micro-Raman. Consequently, for one to characterize the thermal stresses in TSV structures, the bending beam method and micro-Raman can be employed together as complementary techniques.

\section{Acknowledgments}

The authors want to thank Semiconductor Research Corporation and siXis Inc. for the financial support.

\section{References}

[1] Xi Liu, Qiao Chen, P. Dixit, R. Chatterjee, R. Tummala, and S. Sitaraman, "Failure mechanisms and optimum design for electroplated copper Through-Silicon Vias (TSV)," in Electronic Components and Technology Conference, 2009. ECTC 2009. 59th, pp. 624-629, 2009.

[2] S. Ryu, K. H. Lu, X. Zhang, J. Im, P. S. Ho, and R. Huang, "Impact of Near-Surface Thermal Stresses on Interfacial Reliability of Through-Silicon-Vias for 3-D Interconnects," IEEE Transactions on Device and Materials Reliability, vol. 2010.

[3] A. Karmarkar, Xiaopeng Xu, and V. Moroz, "Performanace and reliability analysis of 3D-integration structures employing Through Silicon Via (TSV)," in Reliability Physics Symposium, 2009 IEEE International, pp. 682-687, 2009.

[4] C. Okoro et al., "Extraction of the Appropriate Material Property for Realistic Modeling of Through-Silicon-Vias using $\mu$-Raman Spectroscopy," in Interconnect Technology Conference, 2008. IITC 2008. International, pp. 16-18, 2008.

[5] G. Lee, H. Y. Son, J. K. Hong, K. Y. Byun, and D. Kwon, "Quantification of micropartial residual stress for mechanical characterization of TSV through nanoinstrumented indentation testing," in Electronic Components and Technology Conference (ECTC), 2010 Proceedings 60th, pp. 200-205, 2010.

[6] J. Zhao, T. Ryan, P. S. Ho, A. J. McKerrow, and W. Shih, "On-wafer characterization of thermomechanical properties of dielectric thin films by a bending beam technique," Journal of Applied Physics, 2000.

[7] Y. Du, J. Zhao, and P. Ho, “An Optical Method for Measuring the Two-Dimensional Surface Curvatures of Electronic Packages During Thermal Cycling," Journal of Electronic Packaging, vol. 123, no. 3, pp. 196-199, 2001.

[8] G. G. Stoney, "The Tension of Metallic Films Deposited by Electrolysis," Proceedings of the Royal Society of London. Series A, vol. 82, no. 553, pp. 172-175, 1909.

[9] J. J. Wortman and R. A. Evans, "Young's Modulus, Shear Modulus, and Poisson's Ratio in Silicon and Germanium," Journal of Applied Physics, vol. 36, no. 1, pp. 153-156, 1965.

[10] "Copper - Wikipedia, the free encyclopedia." [Online]. Available: http://en.wikipedia.org/wiki/Copper. [Accessed: 04-Oct-2010].

[11] Zhaozhi Li, Paul Houston, Daniel Baldwin, Gene Stout, Ted Tessier and John Evans, "Processing and Reliability Characterization of a 3D-WLCSP Packaged Component," Proceedings of 59th Electronic Components and Technology Conference, San Diego, CA, pp. 972-979, 2009.

[12] S. P. Baker, R. -. Keller-Flaig, and J. B. Shu, "Bauschinger effect and anomalous thermomechanical deformation induced by oxygen in passivated thin $\mathrm{Cu}$ films on substrates," Acta Materialia, vol. 51, no. 10, pp. 3019-3036, Jun. 2003. 